\title{
Skyrme-Random-Phase-Approximation description of spin-flip and orbital giant resonances
}

\author{
V.O. Nesterenko ${ }^{1}$, J. Kvasil ${ }^{2}$, P. Vesely ${ }^{2,3}$, W. Kleinig ${ }^{1,4}$, and P.-G. Reinhard ${ }^{5}$ \\ ${ }^{1}$ BLTP, Joint Institute for Nuclear Research, \\ 141980, Dubna, Moscow region, Russia \\ nester@theor.jinr.ru; kleinig@theor.jinr.ru \\ ${ }^{2}$ Institute of Particle and Nuclear Physics, \\ Charles University, CZ-18000 Praha, Czech Republic \\ kvasil@ipnp.troja.mff.cuni.cz; vesely@ipnp.troja.mff.cuni.cz \\ ${ }^{3}$ Department of Physics, P.O. Box 35 (YFL), \\ 40014 University of Jyvaskyla, Jyvaskyla, Finland \\ ${ }^{4}$ Technische Universität Dresden, Inst. für Analysis, D-01062, Dresden, Germany. and \\ ${ }^{5}$ Institut für Theoretische Physik II, Universität Erlangen, D-91058, Erlangen, Germany; \\ mpt218@theorie2.physik.uni-erlangen.de
}

(Dated: November 2, 2018)

\begin{abstract}
The self-consistent separable random-phase approximation (SRPA) model with Skyrme forces is extended to the case of magnetic excitations and applied to the description of spin-flip and orbital M1 giant resonances in the isotopic chain ${ }^{142-152} \mathrm{Nd}$. The Skyrme forces SkT6, SkM*, SLy6 and SkI3 are used. The calculations show the onset of the scissors mode with increasing deformation. A specific three-peak structure of the spin-flip response is found and explained by particular neutron and proton spin-flip transitions. Although the employed forces provide an acceptable qualitative description, the Skyrme functional still needs further improvement to reproduce quantitatively the experimental data.
\end{abstract}




\section{INTRODUCTION}

Magnetic giant resonances (GR) represent an important part of the nuclear dynamical response 1]. They were widely investigated within various phenomenological and microscopic models, for surveys see [1, 2, 3, 4]. Now more elaborated Skyrme, Gogny, and relativistic mean-field approaches [5, 6, 7] based on density functional theory (DFT) are at our disposal. They are actively used for the description of nuclear ground states and dynamics covering mainly electrical modes but not magnetic ones. However magnetic modes could be very useful for analyzing and improving these approaches in the parts related to spin densities. Besides, since these modes (especially the spinflip M1 GR) are sensitive to the spin-orbit splitting, they can be used to study the spin-orbit interaction in Skyrme and Gogny forces. They can also help to clarify the role of tensor forces and related spin-orbit densities [8, 9, 10$]$.

This paper is devoted to an investigation of M1 spin-flip and orbital GR within the SkyrmeHartree-Fock (SHF) approach [11, 12, 13]. Earlier SHF studies of these GR were limited to a few explorations [14, 15] and even these were not fully consistent. In the study [14], a hybrid model with partial inclusion of SHF in the Landau-Migdal formulation was exploited while the work [15] used early Skyrme forces and omitted the important spin density. Quite recently a first fully self-consistent systematic SHF investigation of the spin-flip M1 GR was performed [10] within the separable Random-Phase-Approximation (SRPA) model [16, 17] extended to magnetic excitations [10, 18]. It was shown that none of 8 different Skyrme parameterizations was able to describe simultaneously the one-peak structure in doubly-magic nuclei together with the two-peaks in deformed nuclei.

In this paper, the SRPA for magnetic excitations is presented in more detail and applied to an investigation of spin-flip M1 GR in the chain of isotopes ${ }^{142-152} \mathrm{Nd}$. The trends with increasing deformation and the number of neutrons are explored. Besides, we present our first results for the orbital scissors mode [19]. The calculations employ the Skyrme parameterizations SkT6 [20], $\mathrm{SkM}^{*}$ [21], SLy6 [22], and SkI3 [23].

\section{SRPA FOR MAGNETIC EXCITATIONS}

\section{A. General formalism}

SRPA is a fully self-consistent DFT model where both the static mean field and residual interaction are derived from the same functional. The present nuclear application is based on the 
Skyrme functional [11, 12, 13]. It was first derived for electric excitations [16, 17] and then extended to magnetic modes [10, 18]. The self-consistent factorization of the residual interaction in SRPA considerably reduces the computational expense while maintaining a high accuracy. This makes the model very suitable for systematic studies. The SRPA residual interaction includes all contributions from the initial Skyrme functional as well as the Coulomb (direct and exchange) and pairing (at BCS level) terms. The model was widely used for the investigation of electrical GR in spherical and deformed (heavy and super-heavy) nuclei [16, 17, 24, 25].

Starting point is the Skyrme functional with the energy density [5, 7]

$$
\begin{aligned}
\mathcal{H}_{\mathrm{Sk}}= & \frac{b_{0}}{2} \rho^{2}-\frac{b_{0}^{\prime}}{2} \sum_{q} \rho_{q}^{2}+\frac{b_{3}}{3} \rho^{\alpha+2}-\frac{b_{3}^{\prime}}{3} \rho^{\alpha} \sum_{q} \rho_{q}^{2} \\
& +b_{1}\left(\rho \tau-\mathbf{j}^{2}\right)-b_{1}^{\prime} \sum_{q}\left(\rho_{q} \tau_{q}-\mathbf{j}_{q}^{2}\right)-\frac{b_{2}}{2} \rho \Delta \rho+\frac{b_{2}^{\prime}}{2} \sum_{q} \rho_{q} \Delta \rho_{q} \\
& -b_{4}(\rho \nabla \mathbf{J}+(\nabla \times \mathbf{j}) \mathbf{s})-b_{4}^{\prime} \sum_{q}\left(\rho_{q} \nabla \mathbf{J}_{q}+\left(\nabla \times \mathbf{j}_{q}\right) \mathbf{s}_{q}\right) \\
& +\frac{\tilde{b}_{0}}{2} \mathbf{s}^{2}-\frac{\tilde{b}_{0}^{\prime}}{2} \sum_{q} \mathbf{s}_{q}^{2}+\frac{\tilde{b}_{3}}{3} \rho^{\alpha} \mathbf{s}^{2}-\frac{\tilde{b}_{3}^{\prime}}{3} \rho^{\alpha} \sum_{q} \mathbf{s}_{q}^{2}-\frac{\tilde{b}_{2}}{2} \mathbf{s} \cdot \Delta \mathbf{s}+\frac{\tilde{b}_{2}^{\prime}}{2} \sum_{q} \mathbf{s}_{q} \cdot \Delta \mathbf{s}_{q} \\
& +\gamma_{\mathrm{T}}\left(\tilde{b}_{1}\left(\mathbf{s} \cdot \mathbf{T}-\mathbf{J}^{2}\right)+\tilde{b}_{1}^{\prime} \sum_{q}\left(\mathbf{s}_{q} \cdot \mathbf{T}_{q}-\mathbf{J}_{q}^{2}\right)\right)
\end{aligned}
$$

where $\alpha, \gamma_{\mathrm{T}}, b_{i}, b_{i}^{\prime}, \tilde{b}_{i}, \tilde{b}_{i}^{\prime}$ are the force parameters. This functional involves time-even (nucleon $\rho_{q}$, kinetic-energy $\tau_{q}$, spin-orbit $\mathbf{J}_{q}$ ) and time-odd (current $\mathbf{j}_{q}$, spin $\mathbf{s}_{q}$, and vector kinetic-energy $\mathbf{T}_{q}$ ) densities where $q$ denotes protons and neutrons. Densities without index, like $\rho=\rho_{p}+\rho_{n}$, are total. The contributions with $b_{i}(\mathrm{i}=0,1,2,3,4)$ and $b_{i}^{\prime}(\mathrm{i}=0,1,2,3)$ are the standard terms responsible for ground state properties and electric excitations of even-even nuclei [ㅁ, 7]. In the standard SHF, the isovector spin-orbit interaction is linked to the isoscalar one by $b_{4}^{\prime}=b_{4}$. The tensor spin-orbit terms $\propto \gamma_{\mathrm{T}}$ are often skipped. They can be switched in (11) by the parameter $\gamma_{\mathrm{T}}$. The spin terms with $\tilde{b}_{i}, \tilde{b}_{i}^{\prime}$ are relevant for odd nuclei and magnetic modes in even-even nuclei. Though $\tilde{b}_{i}, \tilde{b}_{i}^{\prime}$ may be uniquely determined as functions of $b_{i}, b_{i}^{\prime}$ [7], their values were not yet well tested by nuclear data. As was shown [10], just these spin terms are of a paramount importance for the spin-flip M1.

The general SRPA formalism is given elsewhere [16, 17]. We present here only the basics and peculiarities of magnetic excitations. The SRPA simplifies the residual interaction of Skyrme RPA in a factorized (separable) form as

$$
\hat{V}_{\text {res }}^{\text {sep }}=-\frac{1}{2} \sum_{q q^{\prime}} \sum_{k, k^{\prime}=1}^{K}\left\{\kappa_{q k, q^{\prime} k^{\prime}} \hat{X}_{q k} \hat{X}_{q^{\prime} k^{\prime}}+\eta_{q k, q^{\prime} k^{\prime}} \hat{Y}_{q k} \hat{Y}_{q^{\prime} k^{\prime}}\right\}
$$

where the indices $q$ and $q^{\prime}$ label neutrons and protons, $k$ numbers the separable terms, $\kappa_{q k, q^{\prime} k^{\prime}}$ 
and $\eta_{q k, q^{\prime} k^{\prime}}$ are the corresponding strength matrices, and $\hat{X}_{q k}$ and $\hat{Y}_{q k}$ are time-even and time-odd hermitian one-body operators. We need these two kinds of the operators since the relevant Skyrme functionals involve both time-even and time-odd densities, see [5, 16, 17, 18].

The model uses the Skyrme functional $E\left[J_{q}^{\alpha}(\mathbf{r}, t)\right]=\int d \mathbf{r} \mathcal{H}_{\mathrm{Sk}}(\mathbf{r}, t)$ depending on the local densities $J_{q}^{\alpha} \equiv\left(\rho_{q}, \tau_{q}, \mathbf{J}_{q}, \mathbf{j}_{q}, \mathbf{s}_{q}, \mathbf{T}_{q}\right)$. The separable operators and strength matrices in (2) are selfconsistently derived from this functional and read [16, 17]

$$
\begin{gathered}
\hat{X}_{q k}=\sum_{q^{\prime}} \hat{X}_{q k}^{q^{\prime}}=i \sum_{\alpha^{\prime} \alpha q^{\prime}} \frac{\delta^{2} E}{\delta J_{q^{\prime}}^{\alpha^{\prime}} \delta J_{q}^{\alpha}}\left\langle\left[\hat{P}_{q k}, \hat{J}_{q}^{\alpha}\right]\right\rangle \hat{J}_{q^{\prime}}^{\alpha^{\prime}}, \\
\hat{Y}_{q k}=\sum_{q^{\prime}} \hat{Y}_{q k}^{q^{\prime}}=i \sum_{\alpha^{\prime} \alpha q^{\prime}} \frac{\delta^{2} E}{\delta J_{q^{\prime}}^{\alpha^{\prime}} \delta J_{q}^{\alpha}}\left\langle\left[\hat{Q}_{q k}, \hat{J}_{q}^{\alpha}\right]\right\rangle \hat{J}_{q^{\prime}}^{\alpha^{\prime}}, \\
\kappa_{q^{\prime} k^{\prime}, q k}^{-1}=\sum_{\alpha \alpha^{\prime}} \frac{\delta^{2} E}{\delta J_{q^{\prime}}^{\alpha^{\prime}} \delta J_{q}^{\alpha}}\left\langle\left[\hat{P}_{q k}, \hat{J}_{q}^{\alpha}\right]\right\rangle\left\langle\left[\hat{P}_{q^{\prime} k^{\prime}}, \hat{J}_{q^{\prime}}^{\alpha^{\prime}}\right]\right\rangle, \\
\eta_{q^{\prime} k^{\prime}, q k}^{-1}=\sum_{\alpha \alpha^{\prime}} \frac{\delta^{2} E}{\delta J_{q^{\prime}}^{\alpha^{\prime}} \delta J_{q}^{\alpha}}\left\langle\left[\hat{Q}_{q k}, \hat{J}_{q}^{\alpha}\right]\right\rangle\left\langle\left[\hat{Q}_{q^{\prime} k^{\prime}}, \hat{J}_{q^{\prime}}^{\alpha^{\prime}}\right]\right\rangle .
\end{gathered}
$$

Here $\hat{J}_{q}^{\alpha}$ are the operators associated with the local densities $J_{q}^{\alpha}$. Further, $\hat{Q}_{q k}(\mathbf{r})$ and $\hat{P}_{q k}(\mathbf{r})$ are generalized coordinate (time-even) and momentum (time-odd) hermitian one-body input operators which serve as generators of the separable terms [16, 17]. For E $\lambda$ modes, the input operators $\hat{Q}_{q k}(\mathbf{r})$ are chosen and $\hat{P}_{q k}(\mathbf{r})=i\left[\hat{H}, \hat{Q}_{q k}\right]$ where $\hat{H}$ stands for the full Hamiltonian. Instead, for M $\lambda$ modes, the input operators $\hat{P}_{q k}(\mathbf{r})$ are chosen and $\hat{Q}_{q k}(\mathbf{r})=i\left[\hat{H}, \hat{P}_{q k}\right]$ are deduced.

The RPA equations for coordinate $\bar{q}_{q k}$ and momentum $\bar{p}_{q k}$ variables read

$$
\begin{aligned}
& \sum_{q k}\left\{\bar{q}_{q k}^{\nu}\left[F_{q^{\prime} k^{\prime}, q k}^{(X X)}-\kappa_{q^{\prime} k^{\prime}, q k}^{-1}\right]+\bar{p}_{q k}^{\nu} F_{q^{\prime} k^{\prime}, q k}^{(X Y)}\right\}=0 \\
& \sum_{q k}\left\{\bar{q}_{q k}^{\nu} F_{q^{\prime} k^{\prime}, s k}^{(Y X)}+\bar{p}_{q k}^{\nu}\left[F_{q^{\prime} k^{\prime}, q k}^{(Y Y)}-\eta_{q^{\prime} k^{\prime}, q k}^{-1}\right]\right\}=0
\end{aligned}
$$

with

$$
F_{q^{\prime} k^{\prime}, q k}^{(A B)}=2 \sum_{q^{\prime \prime}, p h \in q^{\prime \prime}} \alpha_{A B} \frac{\left\langle p h \mid \hat{A}_{q k}^{q \prime}\right\rangle^{*}\left\langle p h \mid \hat{B}_{q^{\prime} k^{\prime}}^{q^{\prime \prime}}\right\rangle}{\varepsilon_{p h}^{2}-\omega_{\nu}^{2}}
$$

and

$$
\alpha_{A B}=\left(\begin{array}{ll}
\varepsilon_{p h}, & \text { for } \hat{A}=\hat{B} \\
-i \omega_{\nu}, & \text { for } \hat{A}=\hat{Y}, \hat{B}=\hat{X} \\
i \omega_{\nu}, & \text { for } \hat{A}=\hat{X}, \hat{B}=\hat{Y}
\end{array}\right)
$$


Here $\left\langle p h \mid \hat{A}_{q^{\prime} k^{\prime}}^{q^{\prime \prime}}\right\rangle$ is the matrix element for the two-quasiparticle state $|p h\rangle, \varepsilon_{p h}$ is the energy of this state, $\omega_{\nu}$ is the energy of the RPA state $|\nu\rangle$. The RPA phonon operator reads

$$
\hat{C}_{\nu}^{\dagger}=\sum_{q} \sum_{p h \in q}\left(c_{p h}^{\nu-} \hat{d}_{p h}^{\dagger}-c_{p h}^{\nu+} \hat{d}_{p h}\right)
$$

where $\hat{d}_{p h}^{\dagger}\left(\hat{d}_{p h}\right)$ are the creation (destruction) operators of two-quasiparticle configurations with the amplitudes determined by solutions of (17)-(8):

$$
c_{p h \in q}^{\nu \pm}=-\sum_{q^{\prime} k^{\prime}} \frac{\bar{q}_{q^{\prime} k^{\prime}}^{\nu}\left\langle p h \mid \hat{X}_{q^{\prime} k^{\prime}}^{q}\right\rangle \mp i \bar{p}_{q^{\prime} k^{\prime}}^{\nu}\left\langle p h \mid \hat{Y}_{q^{\prime} k^{\prime}}^{q}\right\rangle}{2\left(\varepsilon_{p h} \pm \omega_{\nu}\right)} .
$$

Following (3)-(6), the separable ansatz (21) explores the residual interaction of the Skyrme functional through the second functional derivatives. The calculations show that, for spin-flip magnetic modes, the spin

$$
\begin{aligned}
\frac{\delta^{2} E}{\delta \mathbf{s}_{q^{\prime}}\left(\mathbf{r}^{\prime}\right) \delta \mathbf{s}_{q}(\mathbf{r})} & =\left[\tilde{b}_{0}-\tilde{b}_{0}^{\prime} \delta_{q q^{\prime}}+\tilde{b}_{3} \frac{2}{3} \rho^{\alpha}(\mathbf{r})-\frac{2}{3} \tilde{b}_{3}^{\prime} \rho^{\alpha}(\mathbf{r}) \delta_{q q^{\prime}}\right. \\
& \left.-\left(\tilde{b}_{2}-\tilde{b}_{2}^{\prime} \delta_{q q^{\prime}}\right) \Delta_{\mathbf{r}}\right] \delta\left(\mathbf{r}-\mathbf{r}^{\prime}\right),
\end{aligned}
$$

spin-orbit

$$
\begin{aligned}
\frac{\delta^{2} E}{\delta \mathbf{J}_{q^{\prime}}\left(\mathbf{r}^{\prime}\right) \delta \rho_{q}(\mathbf{r})} & =\left(b_{4}+b_{4}^{\prime} \delta_{q q^{\prime}}\right) \nabla_{\mathbf{r}} \delta\left(\mathbf{r}-\mathbf{r}^{\prime}\right), \\
\frac{\delta^{2} E}{\delta \mathbf{j}_{k ; q^{\prime}}\left(\mathbf{r}^{\prime}\right) \delta \mathbf{s}_{l ; q}(\mathbf{r})} & =\left(b_{4}+b_{4}^{\prime} \delta_{q q^{\prime}}\right)\left(\varepsilon_{k l m} \nabla_{m ; \mathbf{r}}\right) \delta\left(\mathbf{r}-\mathbf{r}^{\prime}\right),
\end{aligned}
$$

and tensor terms

$$
\begin{aligned}
& \frac{\delta^{2} E}{\delta \mathbf{J}_{q^{\prime}}\left(\mathbf{r}^{\prime}\right) \delta \mathbf{J}_{q}(\mathbf{r})}=-2\left(\tilde{b}_{1}+\tilde{b}_{1}^{\prime} \delta_{q q^{\prime}}\right) \delta\left(\mathbf{r}-\mathbf{r}^{\prime}\right), \\
& \frac{\delta^{2} E}{\delta \mathbf{T}_{q^{\prime}}\left(\mathbf{r}^{\prime}\right) \delta \mathbf{s}_{q}(\mathbf{r})}=\left(\tilde{b}_{1}+\tilde{b}_{1}^{\prime} \delta_{q q^{\prime}}\right) \delta\left(\mathbf{r}-\mathbf{r}^{\prime}\right)
\end{aligned}
$$

are most important. In deformed nuclei, the magnetic modes couple to electric motion [26]. Then the relevant electric terms [16, 17] should be added. For the scissors mode, which exists only in deformed nuclei, the spin-orbit, tensor, and electric terms are essential.

As was mentioned above, the model is self-consistent in the sense that both the static mean field

$$
\hat{h}_{0}=\sum_{\alpha q} \frac{\delta E}{\delta J_{q}^{\alpha}} \hat{J}_{q}^{\alpha}
$$

and the residual interaction (2) are derived from the same functional. The rank of the RPA matrix (77)-(8) is determined by the number $K$ of the input $\hat{Q_{q k}}$ or $\hat{P_{q k}}$ operators. Usually $K=2 \div 5$ 
and so the rank is small [17, 24, 25]. This reduces the computational effort and allows systematic studies even for heavy deformed nuclei.

The pairing functional reads $V_{\text {pair }}=1 / 2 \sum_{q} G_{q} \chi_{q} \chi_{q}^{*}$ where $\chi_{q}$ is the pairing density and $G_{q}$ is

the pairing strength [7]. In the present study, the pairing is included at the BCS level through the quasiparticle energies and Bogoliubov coefficients.

\section{B. Strength function}

GR in heavy nuclei are formed by many RPA states whose detailed structures cannot be resolved experimentally. Then a direct computation of the strength function is more efficient and reasonable. In SRPA the strength function for M1 excitations reads

$$
\begin{aligned}
S(M 1 ; \omega) & =\sum_{\nu \neq 0}\left|\left\langle\Psi_{\nu} \mid \hat{M}\right\rangle\right|^{2} \zeta\left(\omega-\omega_{\nu}\right) \\
& =\Im\left[\frac{\sum_{\beta \beta^{\prime}} F_{\beta \beta^{\prime}}(z) D_{\beta}(z) D_{\beta^{\prime}}(z)}{\pi F(z)}\right]_{z=\omega+i \frac{\Delta}{2}}+\sum_{q, p h \in q}|\langle p h \mid \hat{M}\rangle|^{2} \zeta\left(\omega-\varepsilon_{p h}\right)
\end{aligned}
$$

where $\left\langle\Psi_{\nu} \mid \hat{M}\right\rangle$ is the matrix element of M1 transition between the ground and excited $\left|\Psi_{\nu}\right\rangle$ RPA states, and $\zeta\left(\omega-\omega_{\nu}\right)=\Delta /\left[2 \pi\left[\left(\omega-\omega_{\nu}\right)^{2}+\Delta^{2} / 4\right]\right]$ is the Lorentz weight to simulate the broadening effects beyond SRPA (escape widths, coupling with complex configurations). In the present study, the Lorentz averaging parameter is $\Delta=1 \mathrm{MeV}$. Further, $\beta=q k \tau$ with $\tau$ labeling $X$ and $Y$-operators, $\Im$ means the imaginary part of the value inside the brackets, $F(z)$ is the determinant of the RPA matrix (7)-(8) with $\omega_{\nu}$ replaced by the complex argument $z, F_{\beta \beta^{\prime}}(z)$ is the algebraic supplement of the determinant, and

$$
D_{q k}^{(X)}(z)=\sum_{q^{\prime}, p h \in q^{\prime}} \frac{\omega_{\nu}\left\langle p h \mid X_{q k}^{q^{\prime}}\right\rangle\langle p h \mid \hat{M}\rangle}{\varepsilon_{p h}^{2}-z^{2}}, D_{q k}^{(Y)}(z)=\sum_{q^{\prime}, p h \in q^{\prime}} \frac{i \varepsilon_{p h}\left\langle p h \mid Y_{q k}^{q^{\prime}}\right\rangle\langle p h \mid \hat{M}\rangle}{\varepsilon_{p h}^{2}-z^{2}} .
$$

The operator of M1 transition reads $\hat{M}(M 1 \mu)=\mu_{B} \sqrt{3 /(8 \pi)} \sum_{q}\left[g_{s}^{q} \hat{s}_{\mu}+g_{l}^{q} \hat{l}_{\mu}\right]$ where spin $g$-factors $g_{s}^{p}=5.58 \varsigma$ and $g_{s}^{n}=-3.82 \varsigma$ are quenched by $\varsigma=0.7$ and orbital $g$-factors are $g_{l}^{p}=1$ and $g_{l}^{n}=0$; $\hat{s}_{\mu}$ and $\hat{l}_{\mu}$ are spin and orbital operators.

In the present study, the strength function (18) is calculated only for the branch $\mu=1$ which gives the $K^{\pi}=1^{+}$states relevant for both spin-flip and orbital modes.

\section{Choice of input operators}

The SRPA formalism requires to choose for M1 modes the generating operators $\hat{P}_{q k}(\mathbf{r})$. Their choice is crucial for a fast convergence of the separable expansion $\hat{V}_{\text {res }}^{\text {sep }}$ to the true Skyrme residual 
interaction. We achieve this by using such $\hat{P}_{q k}(\mathbf{r})$ which allow the separable operators $\hat{X}_{q k}(\mathbf{r})$ and $\hat{Y}_{q k}(\mathbf{r})$ to have maxima in different spatial regions of the nucleus, in the surface and interior. Such a flexible choice allows to achieve good convergence already with a few separable terms.

The physical arguments suggest that, for spin-flip mode, the leading input operator $\hat{P}_{q 1}(\mathbf{r})$ should have the form of the spin part of the applied external field (??) with $\mu=1$, i.e. $\hat{P}_{q 1}=\hat{s}_{+}^{q}$. The detailed distributions depends on the interplay of surface and volume excitations. This can be resolved by taking into account the interior of the nucleus [16, 17, 18] by adding input operators with different radial parts, $\hat{P}_{q 2}=r^{2} \hat{s}_{+}^{q}, \hat{P}_{q 3}=r^{4} \hat{s}_{+}^{q}$. This results in $\hat{X}_{q k}(\mathbf{r})$ and $\hat{Y}_{q k}(\mathbf{r})$ interaction operators having more sensitivity in the interior.

In deformed nuclei we should take into account the coupling between magnetic and electric $K^{\pi}=1^{+}$states. So the quadrupole input operator $\hat{Q}_{q}=r^{2} Y_{21}$ with the counterpart $\hat{P}_{q}=i\left[\hat{H}, \hat{Q}_{q}\right]$ should be added. This operator generates quadrupole excitations in both spherical and deformed nuclei. Besides it allows to explore the scissors mode in deformed nuclei.

In Fig. 1, the spin-flip $\left(g_{l}^{n, p}=0\right)$ strength function (18) in ${ }^{150} \mathrm{Nd}$ is plotted for different sets of input operators. It is seen that all the sets give very similar results. In principle, it would suffice to use the minimal set $\hat{P}_{q}=\hat{s}_{+}^{q}$ and $\hat{Q}_{q}=r^{2} Y_{21}$. However, to be on the safe side, we will use in the following calculations a set from 3 input operators, by adding also the operator $\hat{P}_{q}=r^{2} \hat{s}_{+}^{q}$. Then $\mathrm{K}=3$ and we have the RPA matrix of the modest rank $4 \mathrm{~K}=12$.

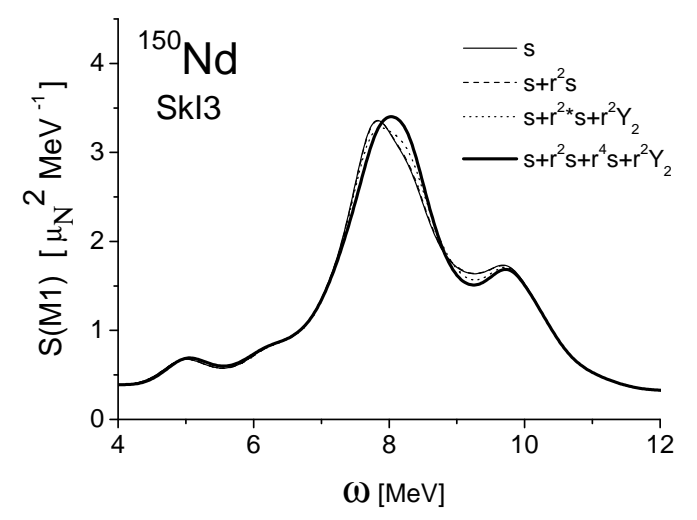

FIG. 1: Strength function for spin-flip M1 GR in ${ }^{150} \mathrm{Nd}$, calculated with the force SkI3 for four sets of input operators, as indicated in the figure.

Note, that in terms of two-quasiparticle matrix elements, the relations between input operators and their counterparts have the form

$$
\hat{P}_{q k} \rightarrow \hat{Q}_{q k}(\mathbf{r})=i\left[\hat{H}, \hat{P}_{q k}\right] \rightarrow\left\langle p h \mid \hat{Q}_{q k}\right\rangle=2 \varepsilon_{p h}\left\langle p h \mid \hat{P}_{q k}\right\rangle-\left\langle p h \mid \hat{X}_{q k}^{q}\right\rangle
$$


for magnetic modes and

$$
\hat{Q}_{q k} \rightarrow \hat{P}_{q k}(\mathbf{r})=i\left[\hat{H}, \hat{Q}_{q k}\right] \rightarrow\left\langle p h \mid \hat{P}_{q k}\right\rangle=2 \varepsilon_{p h}\left\langle p h \mid \hat{Q}_{q k}\right\rangle-\left\langle p h \mid \hat{Y}_{q k}^{q}\right\rangle
$$

for electric modes. For more details see [16, 17, 18].

\section{RESULTS AND DISCUSSION}

Results of the calculations are presented in Figs. 2-4. Fig. 2 shows the spin-flip and orbital M1 strengths for the isotopes ${ }^{142-152} \mathrm{Nd}$. The strengths are computed with the force SkI3 by using $g_{l}^{p}=0$ and $g_{s}^{n, p}=0$, respectively. The isotopic chain ranges from semi-magic spherical $(\mathrm{A}=142)$ to axially deformed $(A=150,152)$ nuclei. The soft nuclei $(A=144,146,148)$ require probably a beyond-RPA treatment. Nevertheless, they are included to illustrate the trends.

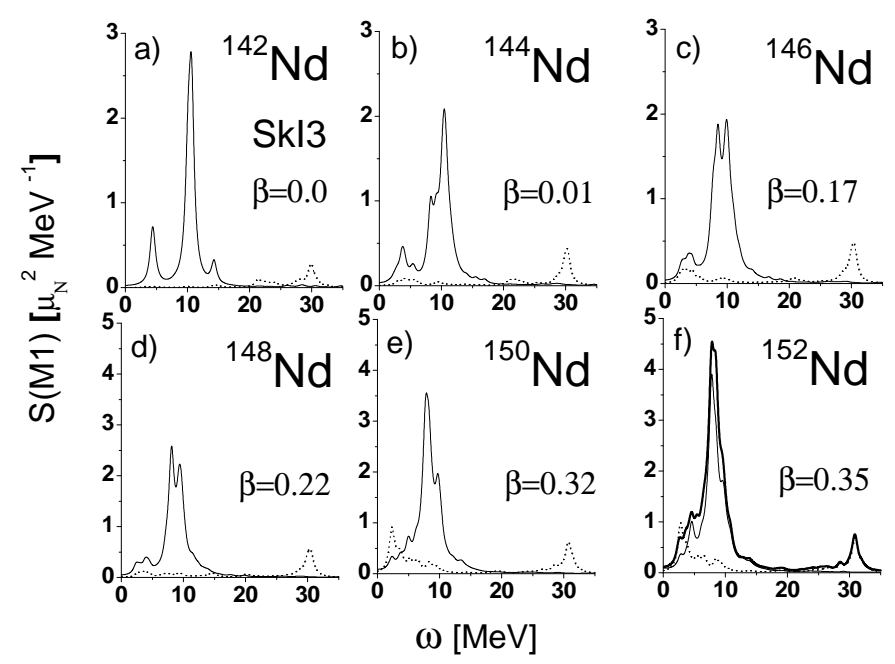

FIG. 2: Spin-flip (solid curve) and orbital (dotted curve) strength functions in ${ }^{142-152} \mathrm{Nd}$, calculated with the force SkI3. For every isotope the parameter of quadrupole deformation $\beta$ defined in Ref. [25] is shown. For ${ }^{152} \mathrm{Nd}$, the total strength (bold curve) with both spin-flip and orbital contributions is presented.

Figure 2 includes the high-energy orbital strength at $30 \mathrm{MeV}$ for all the isotopes. Actually this is the $\lambda \mu=21$ branch of the isovector electric quadrupole GR, though with an overestimated energy. The low-energy scissor orbital mode is absent in spherical ${ }^{142} \mathrm{Nd}$ but then appears and steadily increases with growth of the deformation in heavier isotopes. In deformed ${ }^{150,152} \mathrm{Nd}$, this mode dominates at 2-4 MeV and gives a significant contribution to M1 strength at 2-7 MeV. The comparison of the total, spin-flip and orbital strengths in ${ }^{152} \mathrm{Nd}$ shows that the interference between spin-flip and orbital modes can be both constructive and destructive. The results for other Skyrme forces used in the paper, SkT6, $\mathrm{SkM}^{*}$, and SLy6, are similar. 


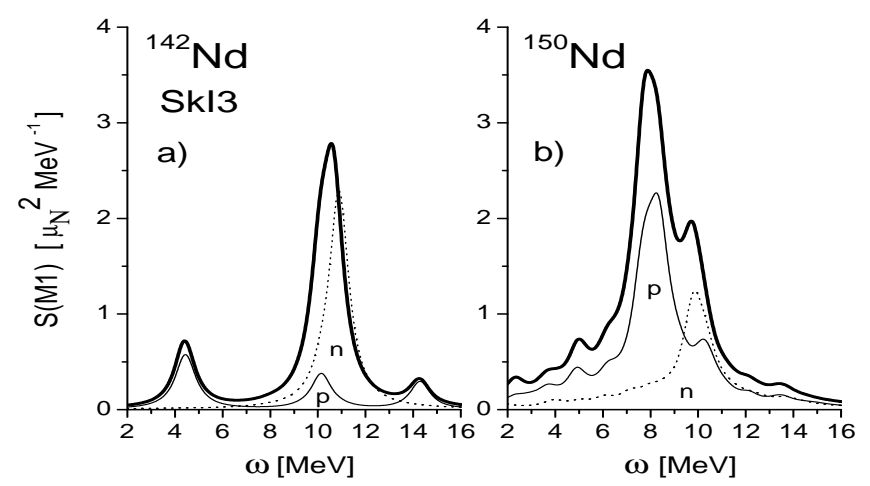

FIG. 3: Total spin-flip (bold curve), proton (solid curve), and neutron (dash curve) strength functions in ${ }^{142} \mathrm{Nd}$ and ${ }^{150} \mathrm{Nd}$, calculated with the force SkI3. The proton and neutron strengths are computed with $g_{s, l}^{n}=0$ and $g_{s, l}^{p}=0$, respectively.

Figure 2 also demonstrates a significant change of the structure of the spin-flip resonance with increasing deformation and neutron number. The distinct two-peak structure at 4 and $10 \mathrm{MeV}$ in ${ }^{142} \mathrm{Nd}$ evolves to a broad one-peak resonance in ${ }^{150,152} \mathrm{Nd}$. Following our analysis of the structure of the RPA states, the spin-flip strength in Nd isotopes is determined by neutron $\nu\left(1 h_{11 / 2}^{-1}, 1 h_{9 / 2}\right)$ and proton $\pi\left(2 d_{5 / 2}^{-1}, 2 d_{3 / 2}\right)$ as well as $\pi\left(1 g_{9 / 2}^{-1}, 1 g_{7 / 2}\right)$ transitions. This is confirmed by Fig. 3 where proton and neutron contributions are separated. One sees that in ${ }^{142} \mathrm{Nd}$ the neutron strength is given by one peak while the proton distribution exhibits two peaks, of $\pi\left(2 d_{5 / 2}^{-1}, 2 d_{3 / 2}\right)$ and $\pi\left(1 g_{9 / 2}^{-1}, 1 g_{7 / 2}\right)$ origin for $4 \mathrm{MeV}$ and $10 \mathrm{MeV}$ structures, respectively. The neutron contribution to the $10 \mathrm{MeV}$ peak weakens from ${ }^{142} \mathrm{Nd}$ to ${ }^{150} \mathrm{Nd}$ since in the latter the neutron subshell $1 h_{9 / 2}$ is already partly occupied. Instead, the proton contribution is concentrated and enforced.

Note that in Fig. 3 the proton and neutron peaks at $10 \mathrm{MeV}$ are close to each other and actually form one broad resonance. This is a consequence of close neutron and proton spin-orbit splittings provided by SkI3 for medium and heavy nuclei [23]. For the comparison, the forces SkM* and SLy6, with more different neutron and proton spin-orbit splittings [10, 21, 22] give a stronger splitting of the resonance in both ${ }^{142} \mathrm{Nd}$ and ${ }^{150} \mathrm{Nd}$ (Fig. 4b,d). Thus the M1 strength distribution is related to the spin-orbit structure of the underlying single-particle states.

The above consideration indicates that the structure of spin-flip M1 mode depends on the Skyrme force. This is further illustrated in Fig. 4 where the results for four Skyrme forces are presented. The forces yield quite different results. Besides, none of these forces provides a proper quantitative description of the experimental data for ${ }^{150} \mathrm{Nd}$ [27]. In particular, the high spike at 6 $\mathrm{MeV}$ is not reproduced. This confirms the finding [10] that most of Skyrme parameterizations do not fully describe the spin-flip spectra. Nevertheless, our calculations allow to suggest a possible 
origin of three M1 peaks observed in nuclides at the onset of rare-earth region [27, 28]. Following the above analysis, they can be considered as $\pi\left(2 d_{5 / 2}^{-1}, 2 d_{3 / 2}\right), \pi\left(1 g_{9 / 2}^{-1}, 1 g_{7 / 2}\right)$, and $\nu\left(1 h_{11 / 2}^{-1}, 1 h_{9 / 2}\right)$ spin-flip transitions.
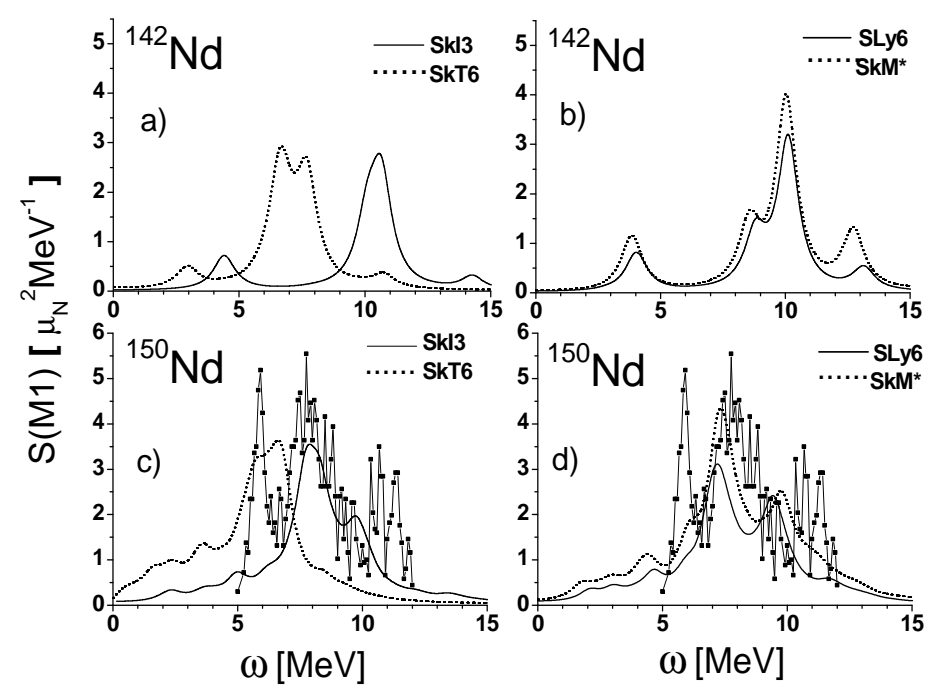

FIG. 4: Total strength function in ${ }^{142} \mathrm{Nd}$ and ${ }^{150} \mathrm{Nd}$ for the Skyrme forces SkT6,SkI3, SkM*, and SLy6. In ${ }^{150} \mathrm{Nd}$ the experimental data [27] are depicted.

\section{CONCLUSIONS}

The orbital and spin-flip M1 giant resonances in Nd isotopes with $\mathrm{A}=142,144,146,148,150$, and 152 were investigated within the self-consistent separable random-phase-approximation model (SRPA) [16, 17]. The calculations show the appearance of the scissors mode while moving from the spherical ${ }^{142} \mathrm{Nd}$ to deformed ${ }^{152} \mathrm{Nd}$. The evolution of the spin-flip strength with deformation and neutron number was analyzed. The observed three-bump structure of this strength is explained by contributions of $\pi\left(2 d_{5 / 2}^{-1}, 2 d_{3 / 2}\right), \pi\left(1 g_{9 / 2}^{-1}, 1 g_{7 / 2}\right)$, and $\nu\left(1 h_{11 / 2}^{-1}, 1 h_{9 / 2}\right)$ spin-flip transitions. It is shown that the largest M1 peak in ${ }^{142} \mathrm{Nd}$ is mainly determined by the neutron contribution. The calculations reveal a still poor quantitative description of spin-flip M1 resonance by present-days Skyrme parameterizations. Further improvement of Skyrme forces is necessary, especially of their spin-orbit parts.

\section{Acknowledgments}

The work was supported by the DFG RE-322/12-1, Heisenberg-Landau (Germany - BLTP JINR), and Votruba - Blokhintsev (Czech Republic - BLTP JINR) grants. W.K. and P.-G.R. are 
grateful for the BMBF support under contracts 06 DD 9052D and 06 ER 9063. Being a part of the research plan MSM 0021620859 (Education Ministry of Czech Republic) this work was also funded by Czech grant agency (grant No. 202/06/0363). P.V. is grateful for the FIDIPRO support.

[1] M.N. Harakeh and A. van der Woude, Giant Resonances (Clarendon Press, Oxford, 2001).

[2] Electric and magnetic giant resonances in nuclei, ed. J. Speth (World Scientific, Singapore, 1991).

[3] F. Osterfeld, Rev. Mod. Phys. 64, 491 (1992).

[4] N. Lo Iudice, Phys. Part. Nucl. 28, 556 (1997).

[5] M. Bender, P.-H. Heenen, and P.-G. Reinhard, Rev. Mod. Phys. 75, 121 (2003).

[6] D. Vretenar, A.V. Afanasjev, G.A. Lalazissis, and P. Ring, Phys. Rep. 409, 101 (2005).

[7] J.R. Stone and P.-G. Reinhard, Prog. Part. Nucl. Phys. 58, 587 (2007).

[8] T. Lesinski et al, Phys. Rev. C 76, 014312 (2007).

[9] W. Zow et al, Phys. Rev. C77, 014314 (2008).

[10] P. Vesely, J. Kvasil, V.O. Nesterenko, W. Kleinig, P.-G. Reinhard, and V.Yu. Ponomarev, Phys. Rev. C80, 031302(R) (2009).

[11] T.H.R. Skyrme, Phil. Mag. 1, 1043 (1956).

[12] D. Vautherin and D.M. Brink, Phys. Rev. C5, 626 (1972).

[13] Y.M. Engel et al, Nucl. Phys. A249, 215 (1975).

[14] P. Sarriguren, E. Moya de Guerra, and R. Nojarov, Phys. Rev. C 54, 690 (1996).

[15] R.R. Hilton, W. Höhenberger, and P. Ring, Eur. Phys. J. A1, 257 (1998).

[16] V.O. Nesterenko, J. Kvasil, and P.-G. Reinhard, Phys. Rev. C 66, 044307 (2002).

[17] V.O. Nesterenko, W. Kleinig, J. Kvasil, P. Vesely, P.-G. Reinhard, and D.S. Dolci, Phys. Rev. C 74, 064306 (2006).

[18] P. Vesely, Ph. D. thesis, Charles University in Prague, Czech Rep., 2009.

[19] N. Lo Iudice and F. Palumbo, Phys. Rev. Lett. 41, 1532 (1978).

[20] F. Tondeur, M. Brack, M. Farine, and J.M. Pearson, Nucl. Phys. A420, 297 (1984).

[21] J. Bartel et al, Nucl. Phys. A386, 79 (1982).

[22] E. Chabanat et al, Nucl. Phys. A627, 710 (1997).

[23] P.-G. Reinhard and H. Flocard, Nucl. Phys. A584, 467 (1995).

[24] V.O. Nesterenko, W. Kleinig, J. Kvasil, P. Vesely, and P.-G. Reinhard, Int. J. Mod. Phys. E 16, 624 (2007); Int. J. Mod. Phys. E 17, 89, (2008).

[25] W. Kleinig, V.O. Nesterenko, J. Kvasil, P.-G. Reinhard, and P. Vesely, Phys. Rev. C 78, 044313 (2008).

[26] J. Kvasil et al, Phys. Rev. C63, 054305 (2001).

[27] H.L. Wörtche, Ph.D. thesis, Technischen Hochschule Darmstadt, Germany, 1994.

[28] D. Frekers et al, Phys. Lett. B244, 178 (1990). 